\title{
Erratum to: Sequestration of arsenate from aqueous solution using 2-line ferrihydrite: equilibria, kinetics, and $\mathrm{X}$-ray absorption spectroscopic analysis
}

\author{
Woo Chun Lee $\cdot$ James Ranville $\cdot$ Seong-Taek Yun •
}

Sun Hee Choi $\cdot$ Soon-Oh Kim

Published online: 19 September 2013

(c) Springer-Verlag Berlin Heidelberg 2013

\section{Erratum to: Environ Earth Sci}

DOI 10.1007/s12665-013-2717-0

The authors would like to correct errors in the original publication as detailed below.

The correct version of the author lists is given here:

Woo Chun Lee · James Ranville $\cdot$ Seong-Taek Yun - Sun Hee Choi $\cdot$ Soon-Oh Kim

The online version of the original article can be found under doi:10.1007/s12665-013-2717-0.

W. C. Lee · S.-O. Kim ( $₫)$

Department of Earth and Environmental Sciences and Research Institute of Natural Science (RINS), Gyeongsang National

University, Jinju 660-701, Republic of Korea

e-mail: sokim@gnu.ac.kr

J. Ranville

Department of Chemistry and Geochemistry, Colorado School

of Mines, Golden, CO 80401, USA

S.-T. Yun

Department of Earth and Environmental Sciences, Korea

University, Seoul 136-701, Republic of Korea

S. H. Choi $(\bowtie)$

Pohang Accelerator Laboratory, Pohang University of Science

and Technology (POSTECH), Pohang 790-784,

Republic of Korea

e-mail: shchoi@postech.ac.kr 